\title{
Contact loci in arc spaces
}

\author{
Lawrence Ein, Robert Lazarsfeld and Mircea Mustaţă
}

\begin{abstract}
We give a geometric description of the loci in the arc space defined by order of contact with a given subscheme, using the resolution of singularities. This induces an identification of the valuations defined by cylinders in the arc space with divisorial valuations. In particular, we recover the description of invariants coming from the resolution of singularities in terms of arcs and jets.
\end{abstract}

\section{Introduction}

The purpose of this paper is to study the loci of arcs on a smooth variety defined by order of contact with a fixed subscheme. Specifically, we establish a Nash-type correspondence showing that the irreducible components of these loci arise from (intersections of) exceptional divisors in a resolution of singularities. We show also that these loci account for all the valuations determined by irreducible cylinders in the arc space. Along the way, we recover in an elementary fashion (without using motivic integration) results of the third author from [Mus01] and [Mus02] relating singularities to arc spaces. Moreover, we extend these results to give a jet-theoretic interpretation of multiplier ideals.

Let $X$ be a smooth complex variety of dimension $d$. Given $m \geqslant 0$ we denote by

$$
X_{m}=\operatorname{Hom}\left(\operatorname{Spec} \mathbb{C}[t] /\left(t^{m+1}\right), X\right)
$$

the space of $m$ th order $\operatorname{arcs}$ on $X$. Thus $X_{m}$ is a smooth variety of dimension $d(m+1)$, and the truncation morphism $\tau_{m+1, m}: X_{m+1} \longrightarrow X_{m}$ realizes each of these spaces as a $\mathbb{C}^{d}$-bundle over the previous one. The inverse limit $X_{\infty}$ of the $X_{m}$ parametrizes all formal arcs on $X$, and one writes $\psi_{m}: X_{\infty} \longrightarrow X_{m}$ for the natural map. These spaces have attracted a great deal of interest in recent years thanks to their central role in the theory of motivic integration (see, for example, [Bat98, DL99, DL98]). In his papers [Mus01] and [Mus02] the third author used this machine to give arc-theoretic interpretations of some of the basic invariants of higher-dimensional geometry.

Consider now a non-zero ideal sheaf $\mathfrak{a} \subseteq \mathcal{O}_{X}$ defining a subscheme $Y \subseteq X$. Given a finite or infinite arc $\gamma$ on $X$, the order of vanishing of $\mathfrak{a}$ (or the order of contact of the corresponding scheme $Y$ ) along $\gamma$ is defined in the natural way. Specifically, pulling $\mathfrak{a}$ back via $\gamma$ yields an ideal $\left(t^{e}\right)$ in $\mathbb{C}[t] /\left(t^{m+1}\right)$ or $\mathbb{C}[[t]]$, and one sets $\operatorname{ord}_{\gamma}(\mathfrak{a})=\operatorname{ord}_{\gamma}(Y)=e$. $\left(\operatorname{Take} \operatorname{ord}_{\gamma}(\mathfrak{a})=m+1\right.$ when $\mathfrak{a}$ pulls back to the zero ideal in $\mathbb{C}[t] /\left(t^{m+1}\right)$ and $\operatorname{ord}_{\gamma}(\mathfrak{a})=\infty$ when it pulls back to the zero ideal in $\mathbb{C}[[t]]$.)

For a fixed integer $p \geqslant 0$, we define the contact loci

$$
\operatorname{Cont}^{p}(Y)=\operatorname{Cont}^{p}(\mathfrak{a})=\left\{\gamma \in X_{\infty} \mid \operatorname{ord}_{\gamma}(\mathfrak{a})=p\right\} .
$$

These are locally closed cylinders, i.e. they arise as the common pull-back of the locally closed sets

$$
\operatorname{Cont}^{p}(Y)_{m}=\operatorname{Cont}^{p}(\mathfrak{a})_{m}=\operatorname{def}\left\{\gamma \in X_{m} \mid \operatorname{ord}_{\gamma}(\mathfrak{a})=p\right\}
$$

Received 20 March 2003, accepted in final form 4 June 2003.

2000 Mathematics Subject Classification 14B05 (primary), 14E15 (secondary).

Keywords: arc space, valuation, log-canonical threshold, multiplier ideals.

This journal is (C) Foundation Compositio Mathematica 2004. 


\section{Ein, R. Lazarsfeld and M. Mustaţă}

defined for any $m \geqslant p$. By an irreducible component $W$ of $\operatorname{Cont}^{p}(\mathfrak{a})$ we mean the inverse image of a component $W_{m}$ of $\operatorname{Cont}^{p}(\mathfrak{a})_{m}$ (these being in bijection for all $m \geqslant p$ via the truncation maps). The codimension of $W$ in $X_{\infty}$ is the common codimension of any of the $W_{m}$ in $X_{m}$.

Our first result establishes a Nash-type correspondence showing that the irreducible components of $\operatorname{Cont}^{p}(\mathfrak{a})$ arise from exceptional divisors appearing in an embedded resolution of $Y$. Specifically, fix a $\log$ resolution $\mu: X^{\prime} \longrightarrow X$ of $\mathfrak{a}$. Thus $X^{\prime}$ is a smooth variety which carries a simple normal crossing divisor $E=\sum_{i=1}^{t} E_{i}, \mu$ is a projective birational map, and

$$
\mathfrak{a}^{\prime}=\mathfrak{a} \cdot \mathcal{O}_{X^{\prime}}=\mathcal{O}_{X^{\prime}}\left(-\sum_{i=1}^{t} r_{i} E_{i}\right) .
$$

For simplicity, we assume here that all $r_{i}$ are positive (we can do this if $\mu$ is an isomorphism over $X \backslash Y$ ), and leave the general case for the main body of the paper. We write

$$
K_{X^{\prime} / X}=\sum_{i=1}^{t} k_{i} E_{i},
$$

where $K_{X^{\prime} / X}=K_{X^{\prime}}-\mu^{*} K_{X}$ is the relative canonical divisor defined by the vanishing of $\operatorname{det} d \mu$. After perhaps some further blowings-up, we may (and do) assume that any non-empty intersection of the $E_{i}$ is connected and hence irreducible. Observe that $\mu$ gives rise in the natural way to morphisms $\mu_{m}: X_{m}^{\prime} \longrightarrow X_{m}$ and $\mu_{\infty}: X_{\infty}^{\prime} \longrightarrow X_{\infty}$.

Fix next a multi-index $\nu=\left(\nu_{i}\right) \in \mathbb{N}^{t}$. We consider the 'multi-contact' locus

$$
\operatorname{Cont}^{\nu}(E)=\left\{\gamma^{\prime} \in X_{\infty}^{\prime} \mid \operatorname{ord}_{\gamma^{\prime}}\left(E_{i}\right)=\nu_{i} \text { for } 1 \leqslant i \leqslant t\right\} .
$$

These are again locally closed cylinders arising from the corresponding subsets $\operatorname{Cont}^{\nu}(E)_{m}$ of $X_{m}^{\prime}$. The philosophy is that these loci can be understood very concretely thanks to the fact that $E$ is a simple normal crossing divisor: for example, $\operatorname{Cont}^{\nu}(E)_{m}$ (if non-empty) is a smooth irreducible variety of codimension $\sum \nu_{i}$ in $X_{m}^{\prime}$.

Our first main result describes the contact loci of $Y$ in terms of the multi-contact loci associated to $E$. In particular, we see that any irreducible component of the contact locus $\operatorname{Cont}^{p}(\mathfrak{a})$ associated to the ideal sheaf $\mathfrak{a}$ on $X$ arises as the image of a unique such multi-contact locus on $X^{\prime}$. This is stated in the following theorem.

Theorem A. For every positive integer $p$, we have a decomposition as a finite disjoint union

$$
\operatorname{Cont}^{p}(\mathfrak{a})=\bigsqcup_{\nu} \mu_{\infty}\left(\operatorname{Cont}^{\nu}(E)\right)
$$

over those $\nu \in \mathbb{N}^{t}$ such that $\sum_{i} \nu_{i} r_{i}=p$. Each $\mu_{\infty}\left(\operatorname{Cont}^{\nu}(E)\right)$ is a constructible cylinder of codimension $\sum_{i} \nu_{i}\left(k_{i}+1\right)$. In particular, for every irreducible component $W$ of $\operatorname{Cont}^{p}(\mathfrak{a})$ there is a unique multi-index $\nu$ as above such that $\operatorname{Cont}^{\nu}(E)$ dominates $W$.

A related result appears in [DL02, Theorem 2.4]. Theorem A yields a quick proof of results of [Mus02] relating the dimensions of the arc spaces $Y_{\ell} \subseteq X_{\ell}$ of $Y$ to the singularities of the pair $(X, Y)$. Keeping notation as above, recall that the log-canonical threshold of $\mathfrak{a}$, or of the pair $(X, Y)$, is the rational number

$$
\operatorname{lct}(\mathfrak{a})=\operatorname{lct}(X, Y)=\operatorname{def}_{i} \min _{i}\left\{\frac{k_{i}+1}{r_{i}}\right\} .
$$

This is an important invariant of the singularities of the functions $f \in \mathfrak{a}$, with smaller values of lct $(\mathfrak{a})$ reflecting nastier singularities (see [DK01, ELSV03, Kol97]). Now consider an irreducible component $V_{\ell}$ of $Y_{\ell}$. Then the inverse image of $V_{\ell}$ in $X_{\infty}$ is contained in the closure of an irreducible component $W$ of the contact locus $\operatorname{Cont}^{p}(Y)$ for some $p \geqslant \ell+1$. Write $\nu=\left(\nu_{i}\right)$ for 
the multi-index describing the cylinder $\operatorname{Cont}^{\nu}(E)$ dominating $W$. If $c=\operatorname{lct}(X, Y)$ then $k_{i}+1 \geqslant c r_{i}$ for each of the divisors $E_{i}$, and so one finds from Theorem A:

$$
\begin{aligned}
\operatorname{codim}\left(V_{\ell}, X_{\ell}\right) & \geqslant \operatorname{codim}\left(\mu_{\infty}\left(\operatorname{Cont}^{\nu}(E)\right), X_{\infty}\right) \\
& =\sum \nu_{i}\left(k_{i}+1\right) \\
& \geqslant c \cdot \sum \nu_{i} r_{i} \\
& =c \cdot p \\
& \geqslant c(\ell+1) .
\end{aligned}
$$

The reverse inequality being elementary for suitable values of $\ell$, we deduce a corollary.

Corollary B [Mus02]. One has

$$
\operatorname{lct}(X, Y)=\min _{\ell}\left\{\frac{\operatorname{codim}\left(Y_{\ell}, X_{\ell}\right)}{\ell+1}\right\} \text {. }
$$

A closer look at this argument identifies geometrically the components of $Y_{\ell}$ having maximal possible dimension: they are the closures of the images of multi-contact loci on $X^{\prime}$ involving the divisors $E_{i}$ computing lct(a) (Corollary 3.3). We also recover the main result of [Mus01] relating irreducibility of $Y_{\ell}$ to the singularities of $Y$ when $Y$ is a local complete intersection.

Our second main result concerns the valuations defined by irreducible cylinders $C \subset X_{\infty}$. Assuming that $C$ does not dominate $X$, it determines a valuation $\operatorname{val}_{C}$ on the function field $\mathbb{C}(X)$ of $X$ by the rule:

$$
\operatorname{val}_{C}(f)=\operatorname{ord}_{\gamma}(f) \quad \text { for general } \gamma \in C .
$$

We prove that any such valuation comes from a contact locus. This is stated in the next theorem.

Theorem C. Let $C \subseteq X_{\infty}$ be an irreducible cylinder which does not dominate $X$, and denote by val $_{C}$ the corresponding valuation on $\mathbb{C}(X)$. Then there exist an ideal $\mathfrak{a} \subseteq \mathcal{O}_{X}$, an integer $p>0$ and an irreducible component $W$ of $\operatorname{Cont}^{p}(\mathfrak{a})$ such that $\operatorname{val}_{C}=\mathrm{val}_{W}$. Moreover val $C$ is a divisorial valuation, i.e. it agrees up to a constant with the valuation given by the order of vanishing along a divisor in a suitable blow-up of $X$.

The divisor in question is the exceptional divisor in a weighted blow-up of $X^{\prime}$ along the intersection of the relevant $E_{i}$ from Theorem A.

Theorem A is a consequence of a result of Denef and Loeser [DL99] describing the fibers of the map $\mu_{m}: X_{m}^{\prime} \longrightarrow X_{m}$. This is also one of the principal inputs to the theory of motivic integration, so the techniques used here are certainly not disjoint from the methods of the third author in [Mus01] and [Mus02]. However the present approach clarifies the geometric underpinnings of the results in question, and it bypasses the combinatorial complexities involved in manipulating motivic integrals.

In order to streamline the presentation, we collect in $\S 1$ some basic results on cylinders in arc spaces of smooth varieties. The proofs of the above theorems are given in $\S 2$. Section 3 is devoted to some variants and further applications: in particular, we complete the proof of Corollary B and explain how to recover some of the results of [Mus01]. We discuss also more general pairs of the form $(X, \alpha \cdot Y-\beta \cdot Z)$, and interpret the corresponding generalized log-canonical thresholds in terms of arc spaces. In particular, this yields a jet-theoretic description of the multiplier ideals. As an example, we treat the case of monomial subschemes in an affine space. 


\section{Ein, R. Lazarsfeld and M. Mustaţă}

\section{Cylinders in arc spaces}

We collect in this section some basic facts on cylinders. Let $X$ be a fixed smooth, connected $d$-dimensional complex variety. We have truncation morphisms $\psi_{m}: X_{\infty} \longrightarrow X_{m}$, and $\tau_{m+1, m}$ : $X_{m+1} \longrightarrow X_{m}$, such that $\tau_{m+1, m}$ is locally trivial with fiber $\mathbb{C}^{d}$. Note that $X_{\infty}$ is the set of $\mathbb{C}$-valued points of a scheme over $\mathbb{C}$, and we consider on $X_{\infty}$ the restriction of the Zariski topology on this scheme. It is clear that this is equal to the inverse limit topology induced by the projections $\left\{\psi_{m}\right\}_{m}$. Since every $\tau_{m+1, m}$ is flat, hence open, it follows that $\psi_{m}$ is open for every $m$.

Recall that a cylinder $C$ in $X_{\infty}$ is a subset of the form $\psi_{m}^{-1}(S)$, for some $m$, and some constructible subset $S \subseteq X_{m}$. We stress that all the points we consider in $X_{\infty}$ and $X_{m}$ are $\mathbb{C}$-valued points. The cylinders form an algebra of sets. It is clear that $C=\psi_{m}^{-1}(S)$ is open (closed, locally closed) if and only if $S$ is. Moreover, we have $\bar{C}=\psi_{m}^{-1}(\bar{S})$, so $\bar{C}$ is a cylinder. Since the projection maps are locally trivial, it follows that $C$ is irreducible if and only if $S$ is. In particular, the decomposition of $S$ in irreducible components induces a similar decomposition for $C$. For every cylinder $C=$ $\psi_{m}^{-1}(S)$, we put $\operatorname{codim}(C):=\operatorname{codim}\left(S, X_{m}\right)=(m+1) d-\operatorname{dim}(S)$. If $C_{1} \subseteq C_{2}$ are cylinders, then $\operatorname{codim}\left(C_{1}\right) \geqslant \operatorname{codim}\left(C_{2}\right)$. Moreover, if $C_{1}$ and $C_{2}$ are closed and irreducible, then we have equality of codimensions if and only if $C_{1}=C_{2}$.

We say that a subset $T$ of $X_{\infty}$ is thin if there is a proper closed subscheme $Z$ of $X$ such that $T \subseteq Z_{\infty}$

Proposition 1.1. If $C$ is a non-empty cylinder, then $C$ is not thin.

Proof. Suppose that $Z$ is a proper closed subset such that $C \subseteq Z_{\infty}$. In particular, we have $\operatorname{codim}(C) \geqslant \operatorname{codim}\left(\psi_{m}^{-1}\left(Z_{m}\right)\right)$ for every $m$. On the other hand, it can be shown that $\lim _{m \rightarrow \infty}$ $\operatorname{codim}\left(\psi_{m}^{-1}\left(Z_{m}\right)\right)=\infty$ (see, for example, [Mus01, Lemma 3.7]). This gives a contradiction.

Lemma 1.2. If $C_{1} \supseteq C_{2} \supseteq C_{3} \supseteq \cdots$ are non-empty cylinders in $X_{\infty}$, then $\bigcap_{m} C_{m} \neq \emptyset$.

Proof. We give a proof following [Bat98]. Note first that a similar assertion holds for a non-increasing sequence of constructible subsets of a given variety, as we work over an uncountable field. Moreover, it follows from definition and Chevalley's theorem that, for every cylinder $C \subseteq X_{\infty}$, the image $\psi_{p}(C)$ is constructible for all $p$.

Consider the constructible subsets of $X$

$$
\psi_{0}\left(C_{1}\right) \supseteq \psi_{0}\left(C_{2}\right) \supseteq \psi_{0}\left(C_{3}\right) \supseteq \cdots .
$$

The above remark shows that we can find $x_{0} \in \bigcap_{m} \psi_{0}\left(C_{m}\right)$. Replace now $C_{m}$ by $C_{m}^{\prime}:=C_{m} \cap \psi_{0}^{-1}\left(x_{0}\right)$, which again form a non-increasing sequence of non-empty cylinders. Consider

$$
\psi_{1}\left(C_{1}^{\prime}\right) \supseteq \psi_{1}\left(C_{2}^{\prime}\right) \supseteq \psi_{1}\left(C_{3}^{\prime}\right) \supseteq \cdots,
$$

so that we can find $x_{1} \in \bigcap_{m} \psi_{m}\left(C_{m}^{\prime}\right)$. We replace now $C_{m}^{\prime}$ by $C_{m}^{\prime \prime}:=C_{m} \cap \psi_{1}^{-1}\left(x_{1}\right)$ and continue this way, to get a sequence $\left\{x_{p}\right\}_{p}$, such that $\tau_{p+1, p}\left(x_{p+1}\right)=x_{p}$ for every $p$, and such that $x_{p} \in \psi_{p}\left(C_{m}\right)$ for every $p$ and $m$. This defines $x=\left(x_{p}\right)_{p} \in X_{\infty}$, and since each $C_{m}$ is a cylinder, we see that $x \in C_{m}$ for every $m$.

We consider now a proper, birational morphism $\mu: X^{\prime} \longrightarrow X$ between smooth varieties, with $\mu_{m}: X_{m}^{\prime} \longrightarrow X_{m}$ the induced map on arc spaces. The next lemma shows that $\mu_{m}$ is surjective for all $m \in \mathbb{N}$. In fact, we will show later the stronger fact that $\mu_{\infty}$ is surjective.

Lemma 1.3. If $\mu$ is as above, then $\mu_{m}: X_{m}^{\prime} \longrightarrow X_{m}$ is surjective for every $m \in \mathbb{N}$.

Proof. Let $Z \subset X$ be a proper closed subset such that $\mu$ is an isomorphism over $X \backslash Z$. It follows from the valuative criterion for properness that $X_{\infty} \backslash Z_{\infty}$ is contained in the image of $\mu_{\infty}$. On the other hand, for every $u \in X_{m}$, we have $\psi_{m}^{-1}(u) \nsubseteq Z_{\infty}$ by Proposition 1.1 , so $u \in \operatorname{Im}\left(\mu_{m}\right)$. 


\section{CONTACT LOCI IN ARC SPACES}

We give now a criterion for the image of a cylinder by $\mu_{\infty}$ to be a cylinder. If $\mu$ is as above, we denote by $\psi_{m}$ and $\psi_{m}^{\prime}$ the projections corresponding to $X$ and $X^{\prime}$, respectively.

Proposition 1.4. With the above notation, if $C^{\prime}=\left(\psi_{m}^{\prime}\right)^{-1}\left(S^{\prime}\right)$ is a cylinder so that $S^{\prime}$ is a union of fibers of $\mu_{m}$, then $C:=\mu_{\infty}\left(C^{\prime}\right)$ is a cylinder in $X_{\infty}$.

Proof. Since $S:=\mu_{m}\left(S^{\prime}\right)$ is constructible, it is enough to show that $C=\psi_{m}^{-1}(S)$. We prove that $C \supseteq \psi_{m}^{-1}(S)$, the reverse inclusion being obvious. If $\gamma \in \psi_{m}^{-1}(S)$, consider for every $p \geqslant m$, the cylinder

$$
D_{p}=\left(\psi_{p}^{\prime}\right)^{-1}\left(\mu_{p}^{-1}\left(\psi_{p}(\gamma)\right)\right) \subseteq X_{\infty}^{\prime} .
$$

It follows from Lemma 1.3 that $D_{p} \neq \emptyset$ for every $p \geqslant m$. On the other hand, it is clear that $D_{p} \supseteq D_{p+1}$ for $p \geqslant m$, so Lemma 1.2 shows that there is $\gamma^{\prime} \in \bigcap_{p \geqslant m} D_{p}$. Note that $\mu_{\infty}\left(\gamma^{\prime}\right)=\gamma$, while we have $\gamma^{\prime} \in C^{\prime}$, as $S^{\prime}$ is a union of fibers of $\mu_{m}$.

We use this to strengthen the assertion in Lemma 1.3.

Corollary 1.5. If $\mu: X^{\prime} \longrightarrow X$ is a proper, birational morphism between smooth varieties, then $\mu_{\infty}$ is surjective.

Proof. $C^{\prime}=X_{\infty}^{\prime}$ certainly satisfies the hypothesis of Proposition 1.4, so $C=\mu_{\infty}\left(X_{\infty}^{\prime}\right)$ is a cylinder. On the other hand, we have seen in the proof of Lemma 1.3 that there is a proper closed subset $Z \subset X$ so that $X_{\infty} \backslash Z_{\infty} \subseteq C$. Therefore $X_{\infty} \backslash C$ is contained in $Z_{\infty}$, so it is empty by Proposition 1.1.

We recall now a theorem of Denef and Loeser which will play a pivotal role in our arguments. Suppose that $\mu: X^{\prime} \longrightarrow X$ is a proper, birational morphism of smooth varieties. As in the Introduction, we introduce the relative canonical divisor

$$
K_{X^{\prime} / X}={ }_{\operatorname{def}}\{\operatorname{det}(d \mu)=0\},
$$

an effective Cartier divisor on $X^{\prime}$, supported on the exceptional locus of $\mu$.

Denef And Loeser Theorem [DL99]. Given an integer $e \geqslant 0$, consider the contact locus

$$
\operatorname{Cont}^{e}\left(K_{X^{\prime} / X}\right)_{m}=\left\{\gamma^{\prime} \in X_{m}^{\prime} \mid \operatorname{ord}_{\gamma^{\prime}}\left(K_{X^{\prime} / X}\right)=e\right\} .
$$

If $m \geqslant 2 e$ then $\operatorname{Cont}^{e}\left(K_{X^{\prime} / X}\right)_{m}$ is a union of fibers of $\mu_{m}: X_{m}^{\prime} \longrightarrow X_{m}$, each of which is isomorphic to an affine space $\mathbb{A}^{e}$. Moreover if

$$
\gamma^{\prime}, \gamma^{\prime \prime} \in \operatorname{Cont}^{e}\left(K_{X^{\prime} / X}\right)_{m}
$$

lie in the same fiber of $\mu_{m}$, then they have the same image in $X_{m-e}^{\prime}$.

In fact, Denef and Loeser show that $\mu_{m}$ is a Zariski-locally trivial $\mathbb{A}^{e}$-bundle over the image of $\operatorname{Cont}^{e}\left(K_{X^{\prime} / X}\right)_{m}$ in $X_{m}$.

Remark 1.6. The most important point for our purposes is the statement that the contact locus Cont $^{e}\left(K_{X^{\prime} / X}\right)_{m} \subseteq X_{m}^{\prime}$ is a union of fibers of $\mu_{m}$, and that all of these fibers are irreducible of dimension $e$. When $\mu: X^{\prime} \longrightarrow X$ is the blowing-up of $X$ along a smooth center this is readily checked by an explicit calculation in local coordinates. This case in turn implies the statement when $\mu$ is obtained as a composition of such blow-ups. There would be no essential loss in generality in limiting ourselves in what follows to such a composition of nice blow-ups.

Corollary 1.7. Let $\mu: X^{\prime} \longrightarrow X$ be a proper, birational morphism between smooth varieties. If $C^{\prime} \subseteq X_{\infty}^{\prime}$ is a cylinder, then the closure $\overline{\mu_{\infty}\left(C^{\prime}\right)}$ of its image is a cylinder. Moreover, if there is $e \in \mathbb{N}$ such that $C^{\prime} \subseteq \operatorname{Cont}^{e}\left(K_{X^{\prime} / X}\right)$, then $\mu_{\infty}\left(C^{\prime}\right)$ is a cylinder. 


\section{Ein, R. Lazarsfeld and M. Mustaţă}

Proof. We use the above Theorem of Denef and Loeser. Note first that, in order to prove the second assertion, we may assume that $C^{\prime}$ is irreducible. Let $e$ be the smallest $p$ such that $C^{\prime} \cap$ $\operatorname{Cont}^{p}\left(K_{X^{\prime} / X}\right) \neq \emptyset$ (we have $e<\infty$ by Proposition 1.1). As $C_{\circ}^{\prime}:=C^{\prime} \cap \operatorname{Cont}^{e}\left(K_{X^{\prime} / X}\right)$ is open and dense in $C^{\prime}$, it follows that $\overline{\mu_{\infty}\left(C^{\prime}\right)}=\overline{\mu_{\infty}\left(C_{\circ}^{\prime}\right)}$, so that it is enough to prove the second assertion in the theorem.

Let $p$ and $T \subseteq X_{p}^{\prime}$ be such that $C^{\prime}=\left(\psi_{p}^{\prime}\right)^{-1}(T)$, and fix $m$ with $m \geqslant \max \{2 e, e+p\}$. If $S \subseteq X_{m}^{\prime}$ is the inverse image of $T$ by the canonical projection to $X_{p}^{\prime}$, Proposition 1.4 shows that it is enough to check that $S$ is a union of fibers of $\mu_{m}$. This follows from Denef and Loeser's theorem: if $\delta_{1} \in S$ and $\delta_{2} \in X_{m}^{\prime}$ are such that $\mu_{m}\left(\delta_{1}\right)=\mu_{m}\left(\delta_{2}\right)$, then the first assertion in the theorem implies $\delta_{2} \in$ Cont $^{e}\left(K_{X^{\prime} / X}\right)_{m}$, and the last assertion in the theorem shows that $\delta_{1}$ and $\delta_{2}$ lie over the same element in $X_{p}^{\prime}$, hence $\delta_{2} \in S$.

We show now that our notion of codimension for cylinders agrees with the usual one, defined in terms of the Zariski topology on $X_{\infty}$.

LEMma 1.8. If $C \subseteq X_{\infty}$ is an irreducible cylinder, and if $W \supseteq C$ is an irreducible closed subset of $X_{\infty}$, then $W$ is a cylinder.

Proof. It follows from the definition of the Zariski topology that $W=\bigcap_{m \in \mathbb{N}} W^{(m)}$, where $W^{(m)}=$ $\psi_{m}^{-1}\left(\overline{\psi_{m}(W)}\right)$. Note that each $W^{(m)}$ is a closed irreducible cylinder, and we have $C \subseteq W^{(m+1)} \subseteq$ $W^{(m)}$ for every $m$. Therefore codim $W^{(m)} \leqslant \operatorname{codim}(C)$ for every $m$, and we deduce that $\operatorname{codim} W^{\left(\frac{m}{}\right)}$ is eventually constant. This shows that there is $m_{0}$ such that $W^{(m)}=W^{\left(m_{0}\right)}$ for all $m \geqslant m_{0}$; hence $W=W^{\left(m_{0}\right)}$ is a cylinder.

Corollary 1.9. If $C \subseteq X_{\infty}$ is a cylinder, then $\operatorname{codim}(C)$ is the codimension of $C$, as defined using the Zariski topology on $X_{\infty}$.

Proof. We may clearly assume that $C$ is closed and irreducible. In this case, the above lemma shows that every chain of closed irreducible subsets containing $C$ consists of cylinders, so the assertion is obvious.

Proposition 1.10. Let $C \subseteq X_{\infty}$ be a cylinder. If we have a countable disjoint union of cylinders $\bigsqcup_{p \in \mathbb{N}} D_{p} \subseteq C$, whose complement in $C$ is thin, then

$$
\operatorname{codim}(C)=\min _{p \in \mathbb{N}} \operatorname{codim}\left(D_{p}\right) .
$$

Moreover, if each $D_{p}$ is irreducible (or empty), then for every irreducible component $W$ of $C$ there is a unique $p \in \mathbb{N}$ such that $D_{p} \subseteq W$, and $D_{p}$ is dense in $W$.

Proof. Let $Z \subset X$ be a proper closed subset such that $C \subseteq Z_{\infty} \cup \bigcup_{p} D_{p}$. We will use the fact that $\lim _{m \rightarrow \infty} \operatorname{codim}\left(\psi_{m}^{-1}\left(Z_{m}\right)\right)=\infty($ see [Mus01, Lemma 3.7]).

It is clear that we have $\operatorname{codim}(C) \leqslant \min _{p} \operatorname{codim}\left(D_{p}\right)$. For the reverse inequality, choose $m$ such that $\operatorname{codim}\left(\psi_{m}^{-1}\left(Z_{m}\right)\right)>\min _{p} \operatorname{codim}\left(D_{p}\right)$. It follows from Lemma 1.2 that there is $r$ such that

$$
C \subseteq \bigcup_{p \leqslant r} D_{p} \cup \psi_{m}^{-1}\left(Z_{m}\right) .
$$

This clearly gives $\operatorname{codim}(C) \geqslant \min _{p} \operatorname{codim}\left(D_{p}\right)$.

Suppose now that every $D_{p}$ is irreducible, and let $W$ be an irreducible component of $C$. Choose $m$ such that $\operatorname{codim}\left(\psi_{m}^{-1}\left(Z_{m}\right)\right)>\operatorname{codim}(W)$, and let $r$ be such that

$$
C \subseteq \bigcup_{p \leqslant r} D_{p} \cup \psi_{m}^{-1}\left(Z_{m}\right)
$$




\section{CONTACT LOCI IN ARC SPACES}

It follows that there is $p \leqslant r$ such that $W \subseteq \overline{D_{p}}$. Since $D_{p}$ is irreducible, we see that we also have $D_{p} \subseteq W$.

Remark 1.11. Under the assumption of Proposition 1.10 one knows that

$$
\mu_{\mathrm{Mot}}(C)=\sum_{p} \mu_{\mathrm{Mot}}\left(D_{p}\right)
$$

where $\mu_{\mathrm{Mot}}$ is the motivic measure from [DL99]. By taking the Hodge realization of the motivic measure, the statement in the above proposition follows immediately. However, we preferred to avoid this formalism.

\section{Contact loci and valuations}

This section is devoted to the proof of our main results. Keeping the notation established in the Introduction, we give first the statement and the proof of a more general version of Theorem A. Consider a smooth variety $X$ of dimension $d$ and a non-zero ideal sheaf $\mathfrak{a} \subseteq \mathcal{O}_{X}$ on $X$ defining a subscheme $Y \subseteq X$. Fix a $\log$ resolution $\mu: X^{\prime} \longrightarrow X$ of $\mathfrak{a}$, with $E=\sum_{i=1}^{t} E_{i}$ a simple normal crossing divisor on $X^{\prime}$ such that

$$
\mathfrak{a}^{\prime}=\mathfrak{a} \cdot \mathcal{O}_{X^{\prime}}=\mathcal{O}_{X^{\prime}}\left(-\sum_{i=1}^{t} r_{i} E_{i}\right), \quad K_{X^{\prime} / X}=\sum_{i=1}^{t} k_{i} E_{i}
$$

for some integers $r_{i}, k_{i} \geqslant 0$. Note that this time we do not assume $r_{i} \geqslant 1$ if $k_{i} \geqslant 1$. Given a multi-index $\nu=\left(\nu_{i}\right) \in \mathbb{N}^{t}$ we define the support of $\nu$ to be

$$
\operatorname{supp}(\nu)=\left\{i \in[1, t] \mid \nu_{i} \neq 0\right\},
$$

and we put

$$
E_{\nu}=\bigcap_{i \in \operatorname{supp}(\nu)} E_{i}
$$

Thus $E_{\nu}$ is either empty or a smooth subvariety of codimension $|\operatorname{supp}(\nu)|$ in $X^{\prime}$. Without loss of generality we will assume in addition that $E_{\nu}$ is connected (and hence irreducible) whenever it is non-empty. We can always arrive at this situation by a sequence of blow-ups along smooth centers. In fact, starting with an arbitrary log resolution, first blow up the $d$-fold intersections of the $E_{i}$; then blow up the $(d-1)$-fold intersections of their proper transforms; and so on. At the end of this process we arrive at a log resolution where the stated condition is satisfied.

Given a multi-index $\nu \in \mathbb{N}^{t}$ and an integer $m \geqslant \max _{i} \nu_{i}$, we consider as in the Introduction the 'multi-contact' loci

$$
\operatorname{Cont}^{\nu}(E)_{m}=\left\{\gamma^{\prime} \in X_{m}^{\prime} \mid \operatorname{ord}_{\gamma^{\prime}}\left(E_{i}\right)=\nu_{i} \text { for } 1 \leqslant i \leqslant t\right\},
$$

and the corresponding sets $\operatorname{Cont}^{\nu}(E) \subseteq X_{\infty}^{\prime}$. Provided that $E_{\nu} \neq \emptyset$, a computation in local coordinates shows that $\operatorname{Cont}^{\nu}(E)_{m}$ is a smooth irreducible locally closed subset of codimension $\sum \nu_{i}$ in $X_{m}^{\prime}$. (If $E_{\nu}$ is empty, then so is $\operatorname{Cont}^{\nu}(E)_{m}$.) Recall that a subset of $X_{\infty}$ is called thin if it is contained in $Z_{\infty}$ for some proper closed subscheme $Z \subset X$.

Theorem 2.1. For every positive integer $p$, we have a disjoint union

$$
\bigsqcup_{\nu} \mu_{\infty}\left(\operatorname{Cont}^{\nu}(E)\right) \subseteq \operatorname{Cont}^{p}(Y)
$$

where the union is over those $\nu \in \mathbb{N}^{t}$ such that $\sum_{i} \nu_{i} r_{i}=p$. For every $\nu$ as above such that $\operatorname{Cont}^{\nu}(E) \neq \emptyset$, its image $\mu_{\infty}\left(\operatorname{Cont}^{\nu}(E)\right)$ is an irreducible cylinder of codimension $\sum_{i} \nu_{i}\left(k_{i}+1\right)$. Moreover, the complement in $\operatorname{Cont}^{p}(Y)$ of the above union is thin. 


\section{Ein, R. Lazarsfeld and M. Mustaţă}

Proof. We use the theorem of Denef and Loeser. It is clear that we have a disjoint union

$$
\bigsqcup_{\nu} \operatorname{Cont}^{\nu}(E) \subseteq \mu_{\infty}^{-1}\left(\operatorname{Cont}^{p}(Y)\right),
$$

where $\nu$ varies over the set in the statement, and the complement is contained in the union of those $\left(E_{i}\right)_{\infty}$ such that $r_{i}=0$. For every $\nu$ we put $e=\sum_{i} \nu_{i} k_{i}$, so that $\operatorname{Cont}^{\nu}(E) \subseteq \operatorname{Cont}^{e}\left(K_{X^{\prime} / X}\right)$. Corollary 1.7 implies that $\mu_{\infty}\left(\operatorname{Cont}^{\nu}(E)\right)$ is a cylinder.

We show now that $\mu_{\infty}\left(\operatorname{Cont}^{\nu}(E)\right)$ and $\mu_{\infty}\left(\operatorname{Cont}^{\nu^{\prime}}(E)\right)$ are disjoint if $\nu \neq \nu^{\prime}$. Indeed, otherwise there are $\gamma \in \operatorname{Cont}^{\nu}(E)$ and $\gamma^{\prime} \in \operatorname{Cont}^{\nu^{\prime}}(E)$ with $\mu_{\infty}(\gamma)=\mu_{\infty}\left(\gamma^{\prime}\right)$. If $e$ and $e^{\prime}$ correspond to $\nu$ and $\nu^{\prime}$, respectively, fix $m \geqslant \max \left\{2 e, e+\nu_{i}, e+\nu_{i}^{\prime}\right\}_{i}$. The first assertion in the theorem of Denef and Loeser gives $e=e^{\prime}$, and the last assertion implies that $\gamma$ and $\gamma^{\prime}$ have the same image in $X_{m-e}^{\prime}$, a contradiction.

Since $\mu_{\infty}$ is surjective by Corollary 1.5, we get a disjoint union as in the theorem. Moreover, if $\nu$ is such that $E_{\nu} \neq \emptyset$ and if $m \gg 0$, then the Theorem of Denef and Loeser shows that the projection

$$
\operatorname{Cont}^{\nu}(E)_{m} \longrightarrow \mu_{m}\left(\operatorname{Cont}^{\nu}(E)_{m}\right)
$$

has irreducible, $e$-dimensional fibers (in fact, it is locally trivial with fiber $\mathbb{A}^{e}$ ). Hence

$$
\operatorname{dim} \mu_{m}\left(\operatorname{Cont}^{\nu}(E)_{m}\right)=\operatorname{dim} \operatorname{Cont}^{\nu}(E)_{m}-e=(m+1) d-\sum_{i} \nu_{i}\left(k_{i}+1\right),
$$

which completes the proof of the theorem.

Remark 2.2. Note that if $\mu$ is as in the introduction, i.e. if it is an isomorphism over $X \backslash Y$, then the disjoint union in Theorem 2.1 is finite. Moreover, it follows from the above proof that in this case the union is equal to $\operatorname{Cont}^{p}(Y)$, and we recover also the first statement of Theorem A.

Remark 2.3. As pointed out by the referee, the fact that

$$
\mu_{\infty}\left(\operatorname{Cont}^{\nu}(E)\right) \cap \mu_{\infty}\left(\operatorname{Cont}^{\nu^{\prime}}(E)\right)=\emptyset
$$

for $\nu \neq \nu^{\prime}$ can be seen also in an elementary way as follows. If $Z=\mu(E)$, then $\mu$ induces an isomorphism $X^{\prime} \backslash E \longrightarrow X \backslash Z$. By the valuative criterion for properness, $\mu_{\infty}$ induces a bijection $X_{\infty}^{\prime} \backslash E_{\infty} \longrightarrow X_{\infty} \backslash Z_{\infty}$. As $\nu_{i}, \nu_{i}^{\prime} \neq \infty$ for all $i$, we see that $\operatorname{Cont}^{\nu}(E)$, $\operatorname{Cont}^{\nu^{\prime}}(E) \subseteq X_{\infty}^{\prime} \backslash E_{\infty}$, and we deduce our assertion.

In the setting of Theorem 2.1, the disjoint decomposition of $\operatorname{Cont}^{p}(Y)$ allows us to relate the irreducible components of $\operatorname{Cont}^{p}(Y)$ with the multi-contact loci $\operatorname{Cont}^{\nu}(E)$. This is a formal consequence of Proposition 1.10.

Corollary 2.4. With the notation in Theorem 2.1, we have

$$
\operatorname{codim} \operatorname{Cont}^{p}(Y)=\min _{\nu} \sum_{i} \nu_{i}\left(k_{i}+1\right),
$$

where the minimum is over all $\nu \in \mathbb{N}^{t}$ such that $\sum_{i} \nu_{i} r_{i}=p$ and $E_{\nu} \neq \emptyset$. Moreover, for every irreducible component $W$ of $\operatorname{Cont}^{p}(Y)$ there is a unique $\nu$ as above, such that $W$ contains $\mu_{\infty}\left(\operatorname{Cont}^{\nu}(E)\right)$ as a dense subset.

Note that this corollary allows us to describe the irreducible components of each $\operatorname{Cont}^{p}(Y)$ which have minimal codimension in terms of the numerical data of the resolution. However, while Theorem 2.1 shows that all the irreducible components are determined by the resolution, the ones of codimension larger than $\operatorname{codim}\left(\operatorname{Cont}^{p}(Y)\right)$ seem to depend on more than just the numerical data. 


\section{CONTACT LOCI IN ARC SPACES}

In the next section we will see that, by considering a varying auxiliary scheme $Z \subset X$, we can describe also other components of the contact loci.

We turn now to valuations of $\mathbb{C}(X)$ associated to cylinders in the arc space. Recall that, if $C$ is an irreducible cylinder in $X_{\infty}$, then we have defined a valuation val $C$ as follows. Note first that if $\gamma \in X_{\infty}$, and if $f$ is a rational function on $X$ defined in a neighborhood of $\psi_{0}(\gamma)$, then $\operatorname{ord}_{\gamma}(f)$ is well defined. If the domain of $f$ intersects $\psi_{0}(C)$, then $\operatorname{val}_{C}(f):=\operatorname{ord}_{\gamma}(f)$, for general $\gamma \in C$. Note that this is a non-negative integer by Proposition 1.1. Since $C$ is irreducible, it follows that $\operatorname{val}_{C}(f)$ is well defined and can be extended to a valuation of the function field of $X$. We will assume from now on that $C$ does not dominate $X$, so val $_{C}$ is non-trivial (and discrete).

It is clear that if $C_{1} \subseteq C_{2}$ are cylinders as above, then $\operatorname{val}_{C_{1}}(f) \geqslant \operatorname{val}_{C_{2}}(f)$ for every rational function $f$ whose domain intersects $\psi_{0}\left(C_{1}\right)$. If, moreover, $C_{1}$ is dense in $C_{2}$, then $\operatorname{val}_{C_{1}}=\operatorname{val}_{C_{2}}$. Suppose now that $\mu: X^{\prime} \longrightarrow X$ is a proper, birational morphism of smooth varieties, and that $C^{\prime} \subseteq X_{\infty}^{\prime}$ is an irreducible cylinder which does not dominate $X^{\prime}$. It follows from Corollary 1.7 that $C:=\overline{\mu_{\infty}\left(C^{\prime}\right)}$ is a cylinder, and we clearly have $\operatorname{val}_{C^{\prime}}=\operatorname{val}_{C}$.

Example 2.5. A divisorial valuation of $\mathbb{C}(X)$ is a discrete valuation associated to a prime divisor on some normal variety $X^{\prime}$ which is birational to $X$. If a divisorial valuation has center on $X$ (see below), then there are infinitely many cylinders in $X_{\infty}$ whose corresponding valuations agree up to a constant with the given valuation. To see this, let $D$ be a prime divisor on $X^{\prime}$, and let $\operatorname{val}_{D}$ be the corresponding valuation. Saying that a valuation has center on $X$ means that we may assume that we have a proper birational morphism $\mu: X^{\prime} \longrightarrow X$, that $X^{\prime}$ is smooth, and that $D$ is smooth on $X^{\prime}$. For $p \geqslant 1$, let $C_{p}^{\prime}=\operatorname{Cont}^{p}(D)$, so $\operatorname{val}_{C_{p}^{\prime}}=p \cdot \operatorname{val}_{D}$. If we put $C_{p}=\overline{\mu_{\infty}\left(C_{p}^{\prime}\right)}$, then Corollary 1.7 shows that $C_{p}$ is an irreducible cylinder, and $\operatorname{val}_{C_{p}}=p \cdot \operatorname{val}_{D}$.

In the remainder of this section we show that, conversely, every valuation defined by a cylinder in $X_{\infty}$ is (up to a constant multiple) a divisorial valuation. We consider first the case when the cylinder is an irreducible component of a contact locus. In this case, the assertion is a corollary of Theorem 2.1.

Corollary 2.6. Let $Y \subset X$ be a proper closed subscheme, and let $W$ be an irreducible component of $\operatorname{Cont}^{p}(Y)$ for some $p \geqslant 1$. Let $\nu \in \mathbb{N}^{t}$ be the multi-index given by Corollary 2.4, so that $\mu_{\infty}\left(\operatorname{Cont}^{\nu}(E)\right)$ is contained and dense in $W$. If $D$ is the exceptional divisor of the weighted blowing-up of $\left(X^{\prime}, E\right)$ with weight $\nu$, then $\operatorname{val}_{W}=q \cdot \operatorname{val}_{D}$, where $q=\operatorname{gcd}\left\{\nu_{i} \mid i \in \operatorname{supp}(\nu)\right\}$.

Proof. Recall the definition of the weighted blowing-up with weight $\nu$. Let

$$
s=\operatorname{lcm}\left\{\nu_{i} \mid i \in \operatorname{supp}(\nu)\right\} .
$$

If $T_{\nu} \subset X^{\prime}$ is the closed subscheme defined by

$$
\sum_{i \in \operatorname{supp}(\nu)} \mathcal{O}\left(-\frac{s}{\nu_{i}} \cdot E_{i}\right),
$$

then the weighted blowing-up of $\left(X^{\prime}, E\right)$ with weight $\nu$ is the normalized blowing-up of $X^{\prime}$ along $T_{\nu}$. There is a unique prime divisor on this blowing-up which dominates $T_{\nu}$; this is $D$.

It follows from our choice of $\nu$ that $\operatorname{val}_{W}=\operatorname{val}_{\operatorname{Cont}^{\nu}(E)}$. Let $g: X^{\prime \prime} \longrightarrow X^{\prime}$ be a proper birational map which factors through the above blowing-up and which satisfies the requirements for a $\log$ resolution. Note that we may consider $D$ as a divisor on $X^{\prime \prime}$. We apply Theorem 2.1 for $g$.

Let $C$ be the multi-contact locus of all $\operatorname{arcs}$ on $X_{\infty}^{\prime \prime}$ with order $q$ along $D$, and order zero along all the other divisors involved. Well-known results about weighted blow-ups show that the coefficient of $D$ in $g^{-1}\left(E_{i}\right)$ is $\nu_{i} / q$, and that the coefficient of $D$ in $K_{X^{\prime \prime}} / X^{\prime}$ is $-1+\sum_{i} \nu_{i} / q$. We see that 


\section{Ein, R. Lazarsfeld And M. Mustaţă}

$g_{\infty}(C) \subseteq \operatorname{Cont}^{\nu}(E)$. Moreover, both these cylinders are irreducible and have the same codimension, as

$$
\operatorname{codim} g_{\infty}(C)=q \cdot \sum_{i} \nu_{i} / q=\operatorname{codim}_{\left(\operatorname{Cont}^{\nu}(E)\right)}
$$

This gives $\operatorname{val}_{W}=\operatorname{val}_{C}=q \cdot \operatorname{val}_{D}$.

We show now that, in fact, we can describe all valuations given by cylinders using contact loci.

Theorem 2.7. If $C$ is an irreducible cylinder in $X_{\infty}$ which does not dominate $X$, then there is a proper closed subscheme $Y \subset X$, a positive integer $p$, and an irreducible component $W$ of $\operatorname{Cont}^{p}(Y)$ such that val $_{C}=$ val $_{W}$. In particular, val $_{C}$ is equal, up to a constant, to a divisorial valuation.

Proof. We have to prove only the first assertion; the second one follows from this and Corollary 2.6. By replacing $C$ with its closure, we may assume that $C$ is closed. Moreover, it is enough to prove our assertion in the case when $X=\operatorname{Spec}(A)$ is affine. Recall that a graded sequence of ideals is a set of ideals $\mathfrak{a}_{\bullet}=\left\{\mathfrak{a}_{p}\right\}_{p \geqslant 1}$ such that $\mathfrak{a}_{p} \cdot \mathfrak{a}_{q} \subseteq \mathfrak{a}_{p+q}$ for all $p$ and $q$ (see [Laz04] for more on this topic). Since $\mathrm{val}_{C}$ is a valuation which is non-negative on $A$, if we define

$$
\mathfrak{a}_{p}:=\left\{f \in A \mid \operatorname{val}_{C}(f) \geqslant p\right\},
$$

then $\mathfrak{a}_{\bullet}$ is a graded sequence of ideals. Note that, since $C$ does not dominate $X$, we have $\mathfrak{a}_{p} \neq(0)$ for every $p$.

Starting with a graded sequence of ideals $\mathfrak{a}_{\bullet}$ as above, we get a sequence of closed cylinders as follows: for every $p \geqslant 1$, let

$$
W_{p}=\left\{\gamma \in X_{\infty} \mid \operatorname{ord}_{\gamma}(f) \geqslant p \text { for every } f \in \mathfrak{a}_{p}\right\} .
$$

Since $\mathfrak{a}_{\bullet}$ is a graded sequence, we have $\mathfrak{a}_{p}^{q} \subseteq \mathfrak{a}_{p q}$, so that $W_{p q} \subseteq W_{p}$ for every $p, q \geqslant 1$. Note that in our case, it follows from definition that $C \subseteq W_{p}$ for all $p$. Moreover, since $\mathfrak{a}_{p} \neq(0)$, we see that $W_{p}$ does not dominate $X$, for any $p$. We put $C_{m}:=W_{m}$ ! so that $C \subseteq C_{m+1} \subseteq C_{m}$ for every $m \geqslant 1$.

We claim that we can choose irreducible components $C_{m}^{\prime}$ of $C_{m}$ such that $C \subseteq C_{m+1}^{\prime} \subseteq C_{m}^{\prime}$ for all $m$. It is clear that for every $m$ we can choose irreducible components $C_{i, m}$ of $C_{i}$ for $i \leqslant m$, so that

$$
C \subseteq C_{m, m} \subseteq C_{m-1, m} \subseteq \cdots \subseteq C_{1, m}
$$

As every cylinder has finitely many irreducible components, there is an irreducible component $C_{1}^{\prime}$ of $C_{1}$ such that $C_{1}^{\prime}=C_{1, m}$ for infinitely many $m$. Similarly, there is an irreducible component $C_{2}^{\prime}$ of $C_{2}$, such that $C_{2}^{\prime} \subseteq C_{1}^{\prime}$, and such that $C_{2}^{\prime}=C_{2, m}$ for infinitely many $m$. Continuing in this way, we deduce our claim.

Note that we have $\operatorname{codim}\left(C_{1}^{\prime}\right) \leqslant \operatorname{codim}\left(C_{2}^{\prime}\right) \leqslant \cdots \leqslant \operatorname{codim}(C)$. Therefore there is $q$ such that $\operatorname{codim}\left(C_{m}^{\prime}\right)=\operatorname{codim}\left(C_{q}^{\prime}\right)$ for every $m \geqslant q$, hence $C_{m}^{\prime}=C_{q}^{\prime}$, as all $C_{i}^{\prime}$ are irreducible closed cylinders. Let $Y$ be the closed subscheme defined by $\mathfrak{a}_{q !}$, and let $\tau:=\min \left\{\operatorname{ord}_{\gamma}(f) \mid \gamma \in C_{q}^{\prime}, f \in \mathfrak{a}_{q !}\right\}$. It is clear that $\tau \geqslant q$ ! and that $C_{q}^{\prime}$ is the closure of an irreducible component of $\operatorname{Cont}^{\tau}(Y)$, so that in order to finish the proof, it is enough to show that val $C=\operatorname{val}_{C_{q}^{\prime}}$.

Since $C \subseteq C_{q}^{\prime}$, we have $\operatorname{val}_{C} \geqslant \operatorname{val}_{C}^{\prime}$ on $A$. Fix $f \in A$, and let us show that $m:=\operatorname{val}_{C}(f) \leqslant$ $\operatorname{val}_{C_{q}^{\prime}}(f)$. By multiplying $f$ with $g$ such that $\operatorname{val}_{C}(g)>0$, we may assume $m \geqslant 1$. Moreover, by taking a suitable power of $f$, we may assume that $m=p$ ! for some $p \geqslant q$. By definition we have $f \in \mathfrak{a}_{m}$, hence

$$
C_{q}^{\prime}=C_{p}^{\prime} \subseteq W_{p !} \subseteq\left\{\gamma \in X_{\infty} \mid \operatorname{ord}_{\gamma}(f) \geqslant m\right\} .
$$

This gives $\operatorname{val}_{C_{q}^{\prime}}(f) \geqslant m$, and completes the proof of the theorem. 


\section{CONTACT LOCI IN ARC SPACES}

Remark 2.8. It follows from Theorem 2.7 that to each irreducible cylinder $C$ which does not dominate $X$ we may associate a (unique) divisor $D$ over $X$ such that $\operatorname{val}_{C}=\lambda \cdot \operatorname{val}_{D}$ for some $\lambda>0$ (of course, we identify two divisors over $X$ which give the same valuation). This map is obviously not injective, but it is surjective by Example 2.5.

Suppose now that $Y \subset X$ is a fixed proper closed subscheme. It would be interesting to understand which divisors appear from the irreducible components of contact loci of $Y$. One can consider this as an embedded version of Nash's problem [Nas95]. If $Y$ is a variety, and if $\psi_{0}: Y_{\infty} \longrightarrow Y$ is the canonical projection, then Nash described an injective map from the set of irreducible components of $\psi_{0}^{-1}\left(Y_{\text {sing }}\right)$ to the set of 'essential' exceptional divisors over $Y$ (divisors which appear in every resolution of $Y$ ). He conjectured that this map is surjective, but a counterexample has been recently found in [IK03].

In the next section we will use Theorem 2.1 to describe certain divisors which are associated to distinguished irreducible components of the contact loci of $Y$, namely the divisors which compute generalized versions of the log-canonical threshold.

\section{Applications to log-canonical thresholds}

We discuss here some applications of Theorem 2.1. As before, $X$ is a smooth irreducible variety of dimension $d, Y \subseteq X$ is a subscheme defined by a non-zero ideal sheaf $\mathfrak{a} \subseteq \mathcal{O}_{X}$, and $\mu: X^{\prime} \longrightarrow X$ is a $\log$ resolution of $(X, Y)$ with

$$
\mathfrak{a} \cdot \mathcal{O}_{X^{\prime}}=\mathcal{O}_{X^{\prime}}\left(-\sum r_{i} E_{i}\right), \quad K_{X^{\prime} / X}=\sum k_{i} E_{i}
$$

\subsection{Arc spaces of subschemes}

We indicate how to recover some of the results of [Mus01] and [Mus02] relating singularities of the pair $(X, Y)$ to the properties of the arc spaces $Y_{\ell} \subseteq X_{\ell}$.

Note that for every irreducible cylinder $C \subseteq X_{\infty}$ there is a subcylinder $C_{0} \subseteq C$ which is open in $C$, such that $\operatorname{ord}_{\gamma}(\mathfrak{a})$ is constant for $\gamma \in C_{0}$. We denote this positive integer by ord $(\mathfrak{a})$ or $\operatorname{ord}_{C}(Y)$. It is clear that, if $p=\operatorname{ord}_{C}(Y)$, then $C$ is contained in the closure of an irreducible component of $\operatorname{Cont}^{p}(Y)$.

Suppose now that $V_{\ell}$ is an irreducible component of $Y_{\ell}$, and let $V=\psi_{\ell}^{-1}\left(V_{\ell}\right) \subseteq X_{\infty}$. Note that $V$ is an irreducible component of

$$
\psi_{\ell}^{-1}\left(Y_{\ell}\right)=\mathrm{Cont}^{\geqslant(\ell+1)}(Y)=_{\operatorname{def}}\left\{\gamma \in X_{\infty} \mid \operatorname{ord}_{\gamma}(Y) \geqslant \ell+1\right\} .
$$

Let $p=\operatorname{ord}_{V}(Y)$, so $p \geqslant \ell+1$. Note that we might have strict inequality (Example 3.1). In any case, $V$ is the closure of an irreducible component of $\operatorname{Cont}^{p}(Y)$. Conversely, the closure of every irreducible component of $\operatorname{Cont}^{p}(Y)$ is the inverse image of an irreducible component of $Y_{p}$. Therefore our analysis of the contact loci $\operatorname{Cont}^{p}(Y)$ gives complete control over the arc spaces of $Y$.

Example 3.1. Let $X=\mathbb{A}^{1}$ with coordinate $t$, and let $Y \subset X$ be defined by $\left(t^{e}\right) \subseteq \mathbb{C}[t]$ for a fixed integer $e \geqslant 2$. Then $V_{1}=Y_{1}$ is the irreducible subset of $X_{1}$ consisting of arcs centered at the origin. However, $\operatorname{ord}_{V}(Y)=e>1$.

We start by completing the proof of Corollary B, namely we prove the following.

Corollary 3.2. The log-canonical threshold of $(X, Y)$ is given by

$$
\operatorname{lct}(X, Y)=\min _{\ell}\left\{\frac{\operatorname{codim}\left(Y_{\ell}, X_{\ell}\right)}{\ell+1}\right\} \text {. }
$$




\section{Ein, R. Lazarsfeld and M. Mustaţă}

Proof. We saw in the Introduction that Theorem A implies the inequality

$$
\operatorname{codim}\left(Y_{\ell}, X_{\ell}\right) \geqslant c(\ell+1)
$$

where $c=\operatorname{lct}(X, Y)$, so it remains only to prove the reverse inequality for suitable $\ell$. But this is immediate. In fact, it follows from the definition of $\operatorname{lct}(X, Y)$ that there exists an index $i$ (say $i=1$ ) for which $k_{1}+1=c r_{1}$. Let $\nu=(1,0,0, \ldots, 0)$ be the multi-index with $\nu_{1}=1$ and $\nu_{i}=0$ for $i>1$. It follows from Theorem 2.1 that $\mu_{\infty}\left(\operatorname{Cont}^{\nu}(E)\right)$ is a subcylinder of $\operatorname{Cont}^{r_{1}}(Y)$ of codimension $k_{1}+1$. If $\ell=r_{1}-1$, then the closure of this subcylinder can be written as $\psi_{\ell}^{-1}(V)$ for some closed subset $V \subseteq Y_{\ell}$, with $\operatorname{codim}\left(V, X_{\ell}\right)=c(\ell+1)$. The first part of the proof implies that $V$ must be an irreducible component of $Y_{\ell}$, so we are done.

The argument just completed leads to an explicit description of the components of $Y_{\ell}$ having maximal possible dimension. Keeping notation as before, let us say that one of the divisors $E_{i} \subseteq X^{\prime}$ computes the log-canonical threshold of $(X, Y)$ if $\operatorname{lct}(X, Y)=\left(k_{i}+1\right) / r_{i}$. Note that in general there may be several divisors $E_{i}$ that compute this threshold.

Corollary 3.3. Let $V_{\ell} \subseteq Y_{\ell}$ be an irreducible component of maximal possible dimension, i.e. with

$$
\operatorname{codim}\left(V_{\ell}, X_{\ell}\right)=(\ell+1) \cdot \operatorname{lct}(X, Y),
$$

and let $V=\psi_{\ell}^{-1}\left(V_{\ell}\right)$ be the corresponding subset of $X_{\infty}$. Then $\operatorname{ord}_{V}(\mathfrak{a})=\ell+1$ and $V$ is dominated by a multi-contact locus $\operatorname{Cont}^{\nu}(E)$ where

$$
\nu_{i} \neq 0 \Longrightarrow E_{i} \text { computes the log-canonical threshold of }(X, Y) .
$$

Conversely the image of any such multi-contact locus $\operatorname{Cont}^{\nu}(E)$ determines a component of $Y_{\ell}$ of maximal possible dimension.

Proof. Write $c=\operatorname{lct}(X, Y)$. We return to the proof of Corollary B. Specifically, $V$ is contained in the closure of some irreducible component $W$ of $\operatorname{Cont}^{p}(\mathfrak{a})$, which in turn is dominated by some multi-contact locus $\operatorname{Cont}^{\nu}(E)$. However, $\operatorname{codim}\left(V_{\ell}, X_{\ell}\right)=c(\ell+1)$ by hypothesis, and hence equality must hold in all the inequalities appearing in Equation (4). Therefore

$$
p=\sum \nu_{i} r_{i}=(\ell+1) \text {. }
$$

Moreover, since in any event $k_{i}+1 \geqslant c r_{i}$ for all $i$, we deduce that $\nu_{i}\left(k_{i}+1\right)=c \nu_{i} r_{i}$ for each index $i$. In particular, if $\nu_{i} \neq 0$ then $E_{i}$ computes the log-canonical threshold of $(X, Y)$. We leave the converse to the reader.

Similar arguments allow one to eliminate motivic integration from the main results of the third author in [Mus01]. For example, we have the following corollary.

Corollary 3.4 [Mus01]. Let $Y \subseteq X$ be a reduced and irreducible locally complete intersection subvariety of codimension $f$. Then the arc space $Y_{\ell}$ is irreducible for all $\ell \geqslant 1$ if and only if $Y$ has at worst rational singularities.

Proof. Following [Mus01] let $\mu: X^{\prime} \longrightarrow X$ be a $\log$ resolution of $(X, Y)$ which dominates the blowing-up of $X$ along $Y$. Write $E_{1}$ for the (reduced and irreducible) exceptional divisor created by this blow-up, so that

$$
r_{1}=1, \quad k_{1}=f-1,
$$

and otherwise keep notation and assumptions as above. It is established in [Mus01], Theorem 2.1 and Remark 2.2 that $Y$ has at worst rational singularities if and only if $k_{i} \geqslant f r_{i}$ for every index $i \geqslant 2$. So we are reduced to showing:

$$
Y_{\ell} \text { is irreducible for all } \ell \geqslant 1 \Longleftrightarrow k_{i} \geqslant f r_{i} \text { for } i \geqslant 2 \text {. }
$$

(It is the proof of this equivalence in [Mus01] that uses motivic integration.) 


\section{CONTACT LOCI IN ARC SPACES}

Assuming that $k_{i} \geqslant f r_{i}$ for $i \geqslant 2$ we show that each $Y_{\ell}$ is irreducible. Note to begin with that $Y_{\ell}$ has one 'main component' $Y_{\ell}^{\text {main }}$, namely the closure of the arc space $\left(Y_{\text {reg }}\right)_{\ell}$ : this component is dominated by the multi-contact locus $\operatorname{Cont}^{(\ell+1,0,0, \ldots, 0)}(E)$ described by the multi-index $\nu=(\ell+$ $1,0,0, \ldots, 0)$. Suppose for a contradiction that there is a further component $V_{\ell}$ of $Y_{\ell}$. In the usual way, $V=\psi_{\ell}^{-1}\left(V_{\ell}\right)$ lies in the closure of an irreducible component $W$ of $\operatorname{Cont}^{p}(Y)$ for $p \geqslant \ell+1$, which via Corollary 2.6 is dominated by a multi-contact locus $\operatorname{Cont}^{\nu}(E)$ for some $\nu=\left(\nu_{i}\right) \neq$ $(\ell+1,0, \ldots, 0)$. Since $Y \subseteq X$ is a local complete intersection of codimension $f$, we have in any event $\operatorname{codim}\left(W, X_{\ell}\right) \leqslant(\ell+1) \cdot f$. In view of the hypothesis $k_{i} \geqslant f r_{i}$ for $i \geqslant 2$ we then find the inequalities

$$
\begin{aligned}
(\ell+1) \cdot f & \geqslant \operatorname{codim}(W) \\
& =\sum_{i \geqslant 1} \nu_{i}\left(k_{i}+1\right) \\
& =\nu_{1} \cdot f+\sum_{i \geqslant 2} \nu_{i}\left(k_{i}+1\right) \\
& \geqslant f \cdot \sum_{i \geqslant 1} \nu_{i} r_{i}+\sum_{i \geqslant 2} \nu_{i} \\
& =f p+\sum_{i \geqslant 2} \nu_{i} .
\end{aligned}
$$

However, since $p \geqslant \ell+1$ this forces $\nu_{i}=0$ for $i \geqslant 2$, a contradiction.

Conversely, suppose that $k_{i}<f r_{i}$ for some $i \geqslant 2$ : say $k_{2} \leqslant f r_{2}-1$. Setting $\nu=(0,1,0, \ldots, 0)$ and $\ell=r_{2}-1$, the multi-contact locus $\operatorname{Cont}^{\nu}(E)$ maps to an irreducible set $W_{\ell} \subseteq Y_{\ell}$ with

$$
\operatorname{codim}\left(W_{\ell}, X_{\ell}\right) \leqslant(\ell+1) f=\operatorname{codim}\left(Y_{\ell}^{\text {main }}, X_{\ell}\right),
$$

and therefore $Y_{\ell}$ cannot be irreducible.

\subsection{Generalized log-canonical thresholds}

We extend now the above results to take into account also an extra scheme $Z$. We fix two proper closed subschemes $Y, Z \subseteq X$ defined by the ideal sheaves $\mathfrak{a}$ and $\mathfrak{b}$, respectively. We use the previous notation for $\mu: X^{\prime} \longrightarrow X$, but this time we assume that $\mu$ is a log resolution for $Y \cup Z$. We write $\mathfrak{b} \cdot \mathcal{O}_{X^{\prime}}=\mathcal{O}_{X^{\prime}}\left(-\sum s_{i} E_{i}\right)$. Having fixed also $\beta \in \mathbb{Q}_{+}$, we define the log-canonical threshold $\operatorname{lct}(X, Y ; \beta \cdot Z)$ to be the largest $\alpha \in \mathbb{Q}_{+}$such that $(X, \alpha \cdot Y-\beta \cdot Z)$ is $\log$ canonical, i.e. $k_{i}+1 \geqslant \alpha \cdot r_{i}-\beta \cdot s_{i}$ for all $i$. Therefore

$$
\operatorname{lct}(X, Y ; \beta \cdot Z)=\min _{i}\left\{\frac{k_{i}+1+\beta \cdot s_{i}}{r_{i}}\right\} .
$$

It is standard to see that the definition does not depend on the particular log resolution [Kol97].

The following corollary is a generalization of Corollary 3.2 to this setting. This time we state the formula in terms of the contact loci, and leave the corresponding statement in terms of arc spaces to the reader.

Corollary 3.5. We have

$$
\operatorname{lct}(X, Y ; \beta \cdot Z)=\min _{C}\left\{\frac{\operatorname{codim}(C)+\beta \cdot \operatorname{ord}_{C}(Z)}{\operatorname{ord}_{C}(Y)}\right\},
$$

where $C$ runs over the irreducible cylinders in $X_{\infty}$ which do not dominate $X$; in fact, it is enough to let $C$ run only over the irreducible components of $\operatorname{Cont}^{p}(Y)$, for $p \geqslant 1$.

Proof. Let $c=\operatorname{lct}(X, Y ; \beta \cdot Z)$. We show first that, if $C$ is an irreducible cylinder in $X_{\infty}$, then $\operatorname{codim}(C) \geqslant c \cdot \operatorname{ord}_{C}(Y)-\beta \cdot \operatorname{ord}_{C}(Z)$. Let $p=\operatorname{ord}_{C}(Y)$, and let $C_{0} \subseteq C$ be an open subcylinder 


\section{Ein, R. Lazarsfeld and M. Mustaţă}

such that $C_{0} \subseteq \operatorname{Cont}^{p}(Y)$. Let $W$ be an irreducible component of $\operatorname{Cont}^{p}(Y)$ containing $C_{0}$, and let $\nu$ be the multi-index corresponding to $W$ by Corollary 2.4. We have

$$
\begin{aligned}
\operatorname{codim}(C) & =\operatorname{codim}\left(C_{0}\right) \\
& \geqslant \operatorname{codim}(W) \\
& =\sum \nu_{i}\left(k_{i}+1\right) \\
& \geqslant \sum_{i} \nu_{i}\left(c \cdot r_{i}-\beta \cdot s_{i}\right) \\
& =c \cdot p-\beta \cdot \operatorname{ord}_{W}(Z) \\
& \geqslant c \cdot p-\beta \cdot \operatorname{ord}_{C}(Z),
\end{aligned}
$$

as required.

In order to finish the proof, it is enough to show that there is $p \geqslant 1$ and an irreducible component

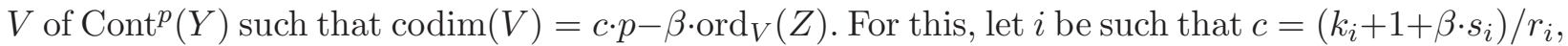
and take $p=r_{i}$ and $\nu$ such that $\nu_{i}=1$ and $\nu_{j}=0$ if $j \neq i$. We may take $V$ to be the closure of $\mu_{\infty}\left(\operatorname{Cont}^{\nu}(E)\right)$ in $\operatorname{Cont}^{p}(Y)$.

We have a similar generalization of Corollary 3.3. Again, we phrase the result in terms of irreducible components of contact loci of $Y$. We say that a divisor $E_{i} \operatorname{computes} \operatorname{lct}(X, Y ; \beta \cdot Z)$ if $\operatorname{lct}(X, Y ; \beta \cdot Y)=\left(k_{i}+1+\beta \cdot s_{i}\right) / r_{i}$.

Corollary 3.6. Let $W$ be an irreducible component of $\operatorname{Cont}^{p}(Y)$, with $p \geqslant 1$, such that

$$
\operatorname{codim}(W)=p \cdot \operatorname{lct}(X, Y ; \beta \cdot Z)-\beta \cdot \operatorname{ord}_{W}(Z) .
$$

Then $W$ is dominated by a multi-contact locus $\operatorname{Cont}^{\nu}(E)$ where

$$
\nu_{i} \neq 0 \quad \Longrightarrow \quad E_{i} \text { computes } \operatorname{lct}(X, Y ; \beta \cdot Z) .
$$

Conversely, the image of any such multi-contact locus determines an irreducible component of $\operatorname{Cont}^{p}(Y)$ as above.

The proof is similar to that of Corollary 3.3 , so we omit it.

Remark 3.7. Recall that we have defined in the previous section a map from the irreducible components of $\operatorname{Cont}^{p}(Y)$ with $p \geqslant 1$ to the divisors over $X$. One can interpret the above corollary as saying that, for every $Z$ and $\beta$, every divisor $D$ which computes $\operatorname{lct}(X, Y ; \beta \cdot Z)$ is in the image of this map. Moreover, the irreducible components which correspond to these divisors are precisely the ones satisfying (8).

Remark 3.8. Suppose that $X=\operatorname{Spec}(A)$ is affine, that $Y$ is defined by the ideal $\mathfrak{a} \subset A$, and that $Z$ is defined by a principal ideal $(f)$. It follows from definition that $\lambda<\operatorname{lct}(X, Y ; Z)$ if and only if $f \in \mathcal{I}(X, \lambda \cdot \mathfrak{a}$ ), the multiplier ideal of $\mathfrak{a}$ with coefficient $\lambda$ (we refer to [Laz04] for the basics on multiplier ideals). One can therefore interpret Corollary 3.5 as giving an arc-theoretic interpretation of multiplier ideals.

We end with an example: monomial ideals in the polynomial ring. For a non-zero monomial ideal $\mathfrak{a}$ in the polynomial ring $R=\mathbb{C}\left[T_{1}, \ldots, T_{d}\right]$, the Newton polyhedron of $\mathfrak{a}$, denoted by $P_{\mathfrak{a}}$, is the convex hull in $\mathbb{R}^{n}$ of the set $\left\{\mathbf{u} \in \mathbb{N}^{d} \mid T^{\mathbf{u}} \in \mathfrak{a}\right\}$. For $\mathbf{u}=\left(\mathbf{u}_{i}\right) \in \mathbb{N}^{d}$, we use the notation $T^{\mathbf{u}}=\prod_{i} T_{i}^{\mathbf{u}_{i}}$. 


\section{CONTACT LOCI IN ARC SPACES}

Proposition 3.9. Let $X=\mathbb{A}^{d}$, and $Y, Z \hookrightarrow X$ subschemes defined by non-zero monomial ideals $\mathfrak{a}$ and $\mathfrak{b} \subseteq R$, respectively, where $R=\mathbb{C}\left[T_{1}, \ldots, T_{d}\right]$. For every $\alpha, \beta \in \mathbb{Q}_{+}$, with $\alpha \neq 0$, we have $(X, \alpha \cdot Y-\beta \cdot Z) \log$-canonical if and only if

$$
\beta \cdot P_{\mathfrak{b}}+\mathbf{e} \subseteq \alpha \cdot P_{\mathfrak{a}},
$$

where $\mathbf{e}=(1, \ldots, 1)$.

Proof. For every $\mathbf{q} \in \mathbb{N}^{d}$, consider the multi-contact locus $C_{\mathbf{q}} \subseteq X_{\infty}$, consisting of those arcs with order $\mathbf{q}_{i}$ along the divisor defined by $T_{i}$, for all $i$. Every contact locus of $Y$ is a union of such cylinders. It follows from Corollary 3.5 that $(X, \alpha \cdot Y-\beta \cdot Z)$ is $\log$ canonical if and only if

$$
\beta \cdot \operatorname{ord}_{C_{\mathbf{q}}}(Z) \geqslant \alpha \cdot \operatorname{ord}_{C_{\mathbf{q}}}(Y)-\operatorname{codim}\left(C_{\mathbf{q}}\right),
$$

for every $\mathbf{q}$.

It is easy to see that $\operatorname{codim}\left(C_{\mathbf{q}}\right)=\sum_{i} \mathbf{q}_{i}$. Moreover, it is clear that we have $\operatorname{ord}_{C_{\mathbf{q}}}(Y)=$ $\inf \left\{\sum_{i} \mathbf{u}_{i} \mathbf{q}_{i} \mid \mathbf{u} \in P_{\mathfrak{a}}\right\}$, and a similar formula for $\operatorname{ord}_{C_{\mathbf{q}}}(Z)$. If we consider the dual polyhedron of $P_{\mathfrak{a}}$

$$
P_{\mathfrak{a}}^{\circ}:=\left\{\mathbf{p} \in \mathbb{R}^{d} \mid \sum_{i} \mathbf{p}_{i} \mathbf{u}_{i} \geqslant 1 \text { for all } \mathbf{u} \in P_{\mathfrak{a}}\right\},
$$

then we see that $\operatorname{ord}_{C_{\mathbf{q}}}(Y) \geqslant m$ if and only if $(1 / m) \mathbf{q} \in P_{\mathfrak{a}}^{\circ}$.

Condition (9) then says that for every $\mathbf{q} \in \mathbb{N}^{d}$ and every $m \in \mathbb{N}^{*}$, such that $(1 / m) \mathbf{q} \in P_{\mathfrak{a}}^{\circ}$, we have

$$
\beta / m \cdot \inf _{\mathbf{v} \in P_{\mathfrak{b}}}\left(\sum_{i} \mathbf{v}_{i} \mathbf{q}_{i}\right)+\sum_{i} \mathbf{q}_{i} / m \geqslant \alpha .
$$

Since $P_{\mathfrak{a}}^{\circ}$ is a rational polyhedron, this is the same as saying that for every $\mathbf{q}^{\prime} \in P_{\mathfrak{a}}^{\circ}$, we have

$$
\beta \cdot \inf _{\mathbf{v} \in P_{\mathfrak{b}}}\left(\sum_{i} \mathbf{q}_{i}^{\prime} \mathbf{v}_{i}\right)+\sum_{i} \mathbf{q}_{i}^{\prime} \geqslant \alpha .
$$

Since $\left(P_{\mathfrak{a}}^{\circ}\right)^{\circ}=P_{\mathfrak{a}}$ and $\alpha>0$, the above condition is equivalent to $\beta \cdot P_{\mathfrak{b}}+\mathbf{e} \subseteq \alpha \cdot P_{\mathfrak{a}}$.

Corollary 3.10 [How01]. If $X=\mathbb{A}^{d}$ and $\mathfrak{a} \subseteq R=\mathbb{C}\left[T_{1}, \ldots, T_{d}\right]$ is a non-zero monomial ideal, then for every $\alpha \in \mathbb{Q}_{+}^{*}$, the multiplier ideal of $\mathfrak{a}$ with coefficient $\alpha$ is given by

$$
\mathcal{I}(X, \alpha \cdot \mathfrak{a})=\left(T^{\mathbf{u}} \mid \mathbf{u}+\mathbf{e} \in \operatorname{Int}\left(\alpha \cdot P_{\mathfrak{a}}\right)\right) .
$$

Proof. Note that multiplier ideals of monomial ideals are monomial (for example, because we can find a $\log$ resolution which is equivariant with respect to the standard $\left(\mathbb{C}^{*}\right)^{d}$-action on $\left.\mathbb{A}^{d}\right)$. If $Z$ is the subscheme defined by $T^{\mathbf{u}}$, note that $T^{\mathbf{u}} \in \mathcal{I}(X, \alpha \cdot \mathfrak{a})$ if and only if $(X, \lambda \cdot Y-Z)$ is log canonical for some $\lambda>\alpha$. The assertion of the corollary follows now from the above proposition.

\section{ACKNOWLEDGEMENTS}

We are grateful to François Loeser and Mihnea Popa for useful discussions, and to the referee for his comments on the paper. Research of the first author was partially supported by NSF Grant 0200278. Research of the second author was partially supported by NSF Grant 01-39713. The third author served as a Clay Mathematics Institute Long-Term Prize Fellow while this research was being carried out. 


\section{CONTACT LOCI IN ARC SPACES}

\section{REFERENCES}

Bat98 V. V. Batyrev, Stringy Hodge numbers of varieties with Gorenstein canonical singularities, in Integrable systems and algebraic geometry (Kobe/Kyoto, 1997) (World Scientific, River Edge, NJ, 1998), 1-32.

DK01 J.-P. Demailly and J. Kollár, Semicontinuity of complex singularity exponents and Kähler-Einstein metrics on Fano orbifolds, Ann. Sci. École Norm. Sup. 34 (2001), 525-556.

DL98 J. Denef and F. Loeser, Motivic Igusa zeta function, J. Algebraic Geom. 7 (1998), 505-537.

DL99 J. Denef and F. Loeser, Germs of arcs on singular varieties and motivic integration, Invent. Math. 135 (1999), 201-232.

DL02 J. Denef and F. Loeser, Lefschetz numbers of iterates of the monodromy and truncated arcs, Topology 41 (2002), 1031-1040.

ELSV03 L. Ein, R. Lazarsfeld, K. E. Smith and D. Varolin, Jumping coefficients of multiplier ideals, Preprint (2003), math.AG/0303002.

How01 J. Howald, Multiplier ideals of monomial ideals, Trans. Amer. Math. Soc. 353 (2001), 2665-2671.

IK03 S. Ishii and J. Kollár, The Nash problem on arc families of singularities, Duke Math. J. 120 (2003), 601-620.

Kol97 J. Kollár, Singularities of pairs, in Algebraic geometry, Santa Cruz, 1995, Proc. Sympos. Pure Math., vol. 62 (American Mathematical Society, Providence, RI, 1997), 221-286.

Laz04 R. Lazarsfeld, Positivity in algebraic geometry, in preparation (2004).

Mus01 M. Mustaţă, Jet schemes of locally complete intersection canonical singularities, with an appendix by D. Eisenbud and E. Frenkel, Invent. Math. 145 (2001), 397-424.

Mus02 M. Mustaţă, Singularities of pairs via jet schemes, J. Amer. Math. Soc. 15 (2002), 599-615.

Nas95 J. F. Nash, Jr., Arc structure of singularities, in A celebration of John F. Nash, Jr., Duke Math. J. 81 (1995), 31-38.

Lawrence Ein ein@math.uic.edu

Department of Mathematics, University of Illinois at Chicago, 851 South Morgan Street (M/C 249), Chicago, IL 60607-7045, USA

Robert Lazarsfeld rlaz@umich.edu

Department of Mathematics, University of Michigan, Ann Arbor, MI 48109, USA

Mircea Mustaţă mirceamustata@yahoo.com

Department of Mathematics, Harvard University, One Oxford Street, Cambridge, MA 02138, USA 